\title{
GAUSSIAN CUBATURE AND BIVARIATE POLYNOMIAL INTERPOLATION
}

\author{
YUAN XU
}

\begin{abstract}
Gaussian cubature is used to study bivariate polynomial interpolation based on the common zeros of quasi-orthogonal polynomials.
\end{abstract}

\section{INTRODUCTION}

Let $\Pi_{n}^{d}$ be the set of polynomials of total degree $n$ in $d$ variables, and let $\Pi^{d}$ be the set of all polynomials in $d$ variables. Let $\mathscr{L}$ be a linear functional defined on $\Pi^{d}$. If $\mathscr{L}\left(g^{2}\right)>0$ whenever $g \in \Pi^{d}$ and $g \neq 0$, then $\mathscr{L}$ is called square positive. Throughout this paper, $\mathscr{L}$ is always assumed to be square positive. A linear functional

$$
I_{N}(f)=\sum_{k=1}^{N} \lambda_{k} f\left(\mathbf{x}_{k}\right), \quad \lambda_{k}>0, \mathbf{x}_{k} \in \mathbb{R}^{d},
$$

is called a cubature formula of degree $m$ if $\mathscr{L}(f)=I_{N}(f)$ whenever $f \in \Pi_{m}^{d}$, and $\mathscr{L}\left(f^{*}\right) \neq I_{N}\left(f^{*}\right)$ for at least one $f^{*} \in \Pi_{m+1}^{d}$. For a fixed $m$, it is known that $N$ satisfies the lower bound

$$
N \geq \operatorname{dim} \Pi_{[m / 2]}^{d} .
$$

We shall call the formulae that attain this lower bound Gaussian cubatures of degree $m$.

For $d=1$, the Gaussian quadratures of degree $2 n-1$ are well known (cf. [2]), and those of degree $2 n-2$ seem to be considered only of secondary interest in most books on numerical analysis. However, we refer to [1] and [3] for a discussion of degree- $(2 n-2)$ quadratures and for historical remarks. For $d=2$, the Gaussian cubatures of degree $2 n-1$ exist only in very special cases (Mysovskikh [9], Möller [6]); it is the case of degree $2 n-2$ that becomes interesting. Gaussian cubatures of degree $2 n-2$ are characterized by Schmid [11] and Morrow and Patterson [7]. The knots of these cubatures are the common zeros of quasi-orthogonal polynomials. In some cases, these zeros are given explicitly.

In this paper we consider the connection between Gaussian cubature of degree $2 n-2$ and Lagrange interpolation. Our approach is based on our recent

Received by the editor May 22, 1991 and, in revised form, November 11, 1991.

1991 Mathematics Subject Classification. Primary 65D30, 41 A05.

Key words and phrases. Gaussian cubature, bivariate orthogonal polynomial, common zeros of quasi-orthogonal polynomials, interpolation. 
development of multivariate orthogonal polynomials [17], which are given in $\S 2$ together with other preliminaries. In $\S 3$ we study quasi-orthogonal polynomials, in particular, an explicit formula for $\lambda_{k}$ in (1.1) will be derived. Bivariate Lagrange interpolation based on the zeros of quasi-orthogonal polynomials will be discussed in $\S 4$. The explicit formula for this Lagrange interpolation will be given and its $L^{2}$ convergence will be discussed.

\section{Preliminaries}

Let $\Pi_{n}$ be the space of polynomials of total degree $n$ in two variables, and let $\Pi$ be the space of all polynomials in two variables. We denote by $\mathscr{L}$ a realvalued linear functional defined on $\Pi$ and square positive. Examples include any linear functional expressible as an integral against a positive measure.

Two polynomials $P$ and $Q$ are said to be orthogonal with respect to $\mathscr{L}$ if $\mathscr{L}(P Q)=0$. For each $n \geq 0$, let $V_{n}$ be the set of polynomials of total degree $k$ that are orthogonal to all polynomials in $\Pi_{n-1}$ together with the zero function. Then $V_{n}$ is a vector space of dimension $n+1$. Clearly, the $V_{n}$ 's are mutually orthogonal. In [17], we have studied the orthogonal polynomials of several variables from the point of view of orthogonality being given in terms of $V_{n}$ rather than in terms of a particular basis of $V_{n}$. However, for the study of cubature formula the basis that contains only the monic polynomials

$$
P_{j}^{n}\left(x_{1}, x_{2}\right)=x_{1}^{j} x_{2}^{n-j}+q_{j}^{n}\left(x_{1}, x_{2}\right), \quad q_{j}^{n} \in \Pi_{n-1}, 0 \leq j \leq n,
$$

seems to be the most convenient one. We shall write

$$
\mathbb{P}_{n}(\mathbf{x})=\left[P_{0}^{n}(\mathbf{x}), P_{1}^{n}(\mathbf{x}), \ldots, P_{n}^{n}(\mathbf{x})\right]^{T}, \quad \mathbf{x}=\left(x_{1}, x_{2}\right) .
$$

For convenience, we sometimes call $\mathbb{P}_{n}$ orthogonal polynomials.

Throughout this paper, the notation $A: i \times j$ means that $A$ is a matrix of size $i \times j$. The $n \times n$ identity matrix is denoted by $I_{n}$. We denote by $A_{n, i}: n \times(n+1), i=1,2$, the matrices

$$
A_{n, 1}=\left[I_{n} \mid 0\right], \quad A_{n, 2}=\left[0 \mid I_{n}\right] .
$$

For a matrix $P=\left(p_{i j}\right)$ whose coefficients $p_{i j}$ belong to $\Pi$, we denote by $\mathscr{L}(P)$ the matrix whose coefficients are the numbers $\mathscr{L}\left(p_{i j}\right)$. For $\mathbb{P}_{n}$ in (2.2) we define

$$
H_{n}=\mathscr{L}\left(\mathbb{P}_{n} \mathbb{P}_{n}^{T}\right)
$$

Since our $\mathscr{L}$ is a square positive linear functional, $H_{n}$ is invertible for all $n \geq 0$.

We now list the properties of the orthogonal polynomials that will be needed later. Most of these properties are proved in [17] for $\mathbb{R}^{d}, d \geq 2$.

$1^{\circ}$. Three-term recurrence relation:

$$
x_{i} \mathbb{P}_{n}=A_{n+1, i} \mathbb{P}_{n+1}+B_{n, i} \mathbb{P}_{n}+H_{n+1} A_{n, i}^{T} H_{n}^{-1} \mathbb{P}_{n-1}, \quad i=1,2,
$$

where $\mathbb{P}_{-1}=0$ and $B_{n, i} H_{n}=\mathscr{L}\left(x_{i} \mathbb{P}_{n} \mathbb{P}_{n}^{T}\right)$.

This relation can be used to give a characterization of orthogonality [17]. Let $K_{n}(\cdot, \cdot)$ denote the reproducing kernel

$$
K_{n}(\mathbf{x}, \mathbf{y})=\sum_{k=0}^{n-1} \mathbb{P}_{k}^{T}(\mathbf{x}) H_{k}^{-1} \mathbb{P}_{k}(\mathbf{y}), \quad \mathbf{x}, \mathbf{y} \in \mathbb{R}^{2}
$$


$2^{\circ}$. For an arbitrary point $\mathbf{t}$ in $\mathbb{R}^{2}$,

$$
K_{n}(\mathbf{t}, \mathbf{t})^{-1}=\min \mathscr{L}\left(P^{2}\right),
$$

where the minimum is over all polynomials in $\Pi_{n-1}$ subject to the condition $P(\mathbf{t})=1$.

$3^{\circ}$. Christoffel-Darboux formula:

$$
K_{n}(\mathbf{x}, \mathbf{y})=\frac{\left[A_{n, i} \mathbb{P}_{n}(\mathbf{x})\right]^{T} H_{n-1}^{-1} \mathbb{P}_{n-1}(\mathbf{y})-\mathbb{P}_{n-1}^{T}(\mathbf{x}) H_{n-1}^{-1}\left[A_{n, i} \mathbb{P}_{n}(\mathbf{y})\right]}{x_{i}-y_{i}},
$$

$i=1,2$, where $\mathbf{x}=\left(x_{1}, x_{2}\right)$ and $\mathbf{y}=\left(y_{1}, y_{2}\right)$.

For further properties of these orthogonal polynomials, we refer to [10, 14, 17] and the references given there. The reproducing kernel has been used in the study of cubature in Mysovskikh [10] and Möller [5].

To describe the results on Gaussian cubatures of degree $2 n-2$, we need the notion of quasi-orthogonal polynomials. Let $\left\{\mathbb{P}_{n}\right\}_{n=0}^{\infty}$ be a sequence of orthogonal polynomials with respect to $\mathscr{L}$. A sequence of polynomials $\left\{\mathbb{Q}_{n}\right\}_{n=0}^{\infty}$ is called quasi-orthogonal with respect to $\mathscr{L}$, if

$$
\mathbb{Q}_{n}(\mathbf{x})=\mathbb{P}_{n}(\mathbf{x})+\Gamma_{n}^{T} H_{n-1}^{-1} \mathbb{P}_{n-1}(\mathbf{x}),
$$

where $\Gamma_{n}$ is a real Hankel matrix of size $n \times(n+1)$. A matrix $\Gamma$ is called a Hankel matrix if $\Gamma=\left(\gamma_{i+j}\right)$. We note that $\mathbb{Q}_{n}$ is orthogonal to $\Pi_{n-2}$, and the components in $\mathbb{Q}_{n}$ are monic polynomials in $\Pi_{n}$. For the univariate theory of quasi-orthogonal polynomials, we refer to $[1,3]$. The use of quasi-orthogonal polynomials in the study of cubatures has appeared in several papers [7, 11, 14]. The present vector-matrix formulation has been used in [14]. The following theorem was proved by Schmid [11] and Morrow and Patterson [7].

Theorem 2.1. There exists a Gaussian cubature of degree $2 n-2$,

$$
I_{N}(f)=\sum_{k=1}^{N} \lambda_{k n} f\left(\mathbf{x}_{k n}\right), \quad N=\left(\begin{array}{c}
n+1 \\
2
\end{array}\right),
$$

if and only if $\mathbf{x}_{k n}, 1 \leq k \leq N$, are the common zeros of the components in $\mathbb{Q}_{n}$.

We shall call the common zeros of the components in $\mathbb{Q}_{n}$ zeros of $\mathbb{Q}_{n}$. The existence of zeros of $\mathbb{Q}_{n}$ is also considered in [11] and [7], where polynomial ideal theory is used. We list the main results as

Theorem 2.2. Let $\left\{\mathbb{Q}_{n}\right\}$ be a sequence of quasi-orthogonal polynomials. Then $\mathbb{Q}_{n}$ has $N=\operatorname{dim} \Pi_{n-1}$ distinct real zeros if and only if there exists a real matrix $\Lambda_{n}: n \times(n+1)$, such that

$$
x_{2} A_{n, 1} \mathbb{Q}_{n}-x_{1} A_{n, 2} \mathbb{Q}_{n}=\Lambda_{n} \mathbb{Q}_{n} .
$$

There are several equivalent conditions to (2.11). The most constructive one in $[7,11,14]$ states that $\Gamma_{n}$ in $(2.9)$ has to satisfy a nonlinear matrix equation involving the coefficient matrices in (2.5). Unfortunately, this nonlinear matrix equation is quite complicated. It takes the form

$$
\begin{aligned}
A_{n, 1} H_{n} A_{n, 2}^{T}-A_{n, 2} H_{n} A_{n, 1}^{T}= & \Gamma_{n}\left(A_{n, 1}^{T} H_{n-1}^{-1} A_{n, 2}-A_{n, 2}^{T} H_{n-1}^{-1} A_{n, 1}\right) \Gamma_{n}^{T} \\
& -\left[\Gamma_{n}\left(A_{n, 1} B_{n, 2}-A_{n, 2} B_{n, 1}\right)^{T}\right. \\
& \left.-\left(A_{n, 1} B_{n, 2}-A_{n, 2} B_{n, 1}\right) \Gamma_{n}^{T}\right] .
\end{aligned}
$$


If $\mathscr{L}$ is centrally symmetric, i.e., $\mathscr{L}\left(x_{1}^{i} x_{2}^{j}\right)=0$ if $i+j$ is odd, this equation takes a simpler form, since then $B_{n, i}=0$. The solution is found only in some special centrally symmetric cases; see the examples in $\S 4$.

The following properties of Hankel matrices will be used later.

A matrix $\Gamma_{n}: n \times(n+1)$ is a Hankel matrix if and only if

$$
A_{n-1,1} \Gamma_{n} A_{n, 2}^{T}=A_{n-1,2} \Gamma_{n} A_{n, 1}^{T} \text {. }
$$

Furthermore, if $\Gamma_{n}: n \times(n+1)$ is a Hankel matrix, then

$$
A_{n, i} \Gamma_{n}^{T}=\Gamma_{n} A_{n, i}^{T}, \quad i=1,2 .
$$

\section{QUASI-ORTHOGONAL POLYNOMIALS AND CUBATURES}

In this section we prove some properties of quasi-orthogonal polynomials. These properties will be used in the next section to study Lagrange interpolation. An explicit formula for $\lambda_{k n}$ in (2.10) will be derived as well. We begin with

Theorem 3.1. Let $\left\{\mathbb{P}_{n}\right\}_{n=0}^{\infty}$ be the orthogonal polynomials in (2.1). Let $\mathbb{Q}_{n}$ be the quasi-orthogonal polynomials in (2.9). Then

$$
K_{n}(\mathbf{x}, \mathbf{y})=\frac{\left[A_{n, i} \mathbb{Q}_{n}(\mathbf{x})\right]^{T} H_{n-1}^{-1} \mathbb{P}_{n-1}(\mathbf{y})-\mathbb{P}_{n-1}^{T}(\mathbf{x}) H_{n-1}^{-1}\left[A_{n, i} \mathbb{Q}_{n}(\mathbf{y})\right]}{x_{i}-y_{i}},
$$

$i=1,2$, where $K_{n}(\cdot, \cdot)$ is the reproducing kernel of $\left\{\mathbb{P}_{n}\right\}$ defined in (2.6).

Proof. From the Christoffel-Darboux formula (2.8) and the definition of $\mathbb{Q}_{n}$ in (2.9), we have

$$
\begin{aligned}
\left(x_{i}-y_{i}\right) K_{n}(\mathbf{x}, \mathbf{y})= & {\left[A_{n, i} \mathbb{P}_{n}(\mathbf{x})\right]^{T} H_{n-1}^{-1} \mathbb{P}_{n-1}(\mathbf{y})-\mathbb{P}_{n-1}^{T}(\mathbf{x}) H_{n-1}^{-1}\left[A_{n, i} \mathbb{P}_{n}(\mathbf{y})\right] } \\
= & {\left[A_{n, i} \mathbb{Q}_{n}(\mathbf{x})\right]^{T} H_{n-1}^{-1} \mathbb{P}_{n-1}(\mathbf{y})-\mathbb{P}_{n-1}^{T}(\mathbf{x}) H_{n-1}^{-1}\left[A_{n, i} \mathbb{Q}_{n}(\mathbf{y})\right] } \\
& -\left[A_{n, i} \Gamma_{n}^{T} H_{n-1}^{-1} \mathbb{P}_{n-1}^{T}(\mathbf{x})\right]^{T} H_{n-1}^{-1} \mathbb{P}_{n-1}(\mathbf{y}) \\
& +\mathbb{P}_{n-1}^{T}(\mathbf{x}) H_{n-1}^{-1} A_{n, i} \Gamma_{n}^{T} H_{n-1}^{-1} \mathbb{P}_{n}(\mathbf{y}) .
\end{aligned}
$$

Since $\Gamma_{n}$ is a Hankel matrix, it follows from (2.14) that the last two terms cancel each other.

Corollary 3.2. Let $\mathbf{x}_{k n}$ and $\mathbf{x}_{j n}$ be any two distinct common zeros of $\mathbb{Q}_{n}$. Then

$$
K_{n}\left(\mathbf{x}_{k n}, \mathbf{x}_{j n}\right)=0 \text {. }
$$

Proof. Since $\mathbf{x}_{k n}$ and $\mathbf{x}_{j n}$ are distinct, they differ in at least one corresponding coordinate. Therefore, the result follows easily from Theorem 3.1.

Theorem 3.3. Under the assumption of Theorem 3.1,

$$
\begin{aligned}
K_{n}(\mathbf{x}, \mathbf{x})= & \mathbb{P}_{n-1}^{T}(\mathbf{x}) H_{n-1}^{-1} A_{n, i} \partial_{i} \mathbb{Q}_{n}(\mathbf{x}) \\
& -\left[A_{n, i} \mathbb{Q}_{n}(\mathbf{x})\right]^{T} H_{n-1}^{-1} \partial_{i} \mathbb{P}_{n-1}(\mathbf{x}), \quad i=1,2,
\end{aligned}
$$

where $\partial_{i}=\partial / \partial x_{i}$ denotes the partial derivative with respect to $x_{i}$.

Proof. Since $\mathbb{P}_{n-1}^{T}(\mathbf{x}) H_{n-1}^{-1}\left[A_{n, i} \mathbb{Q}_{n}(\mathbf{x})\right]$ is a scalar function, it is equal to its own transpose. Therefore we have from (3.1) that

$$
\begin{aligned}
\left(x_{i}-y_{i}\right) K_{n}(\mathbf{x}, \mathbf{y})= & {\left[A_{n, i} \mathbb{Q}_{n}(\mathbf{x})\right]^{T} H_{n-1}^{-1}\left[\mathbb{P}_{n-1}(\mathbf{y})-\mathbb{P}_{n-1}(\mathbf{x})\right] } \\
& -\mathbb{P}_{n-1}^{T}(\mathbf{x}) H_{n-1}^{-1} A_{n, i}\left[\mathbb{Q}_{n}(\mathbf{y})-\mathbb{Q}_{n}(\mathbf{x})\right] .
\end{aligned}
$$


Thus, (3.3) follows by dividing the above identity by $x_{i}-y_{i}$ and letting $y_{i} \rightarrow$ $x_{i}$.

Remark. We note that the right-hand side of (3.3) is independent of $i$.

If $\mathbf{x}^{*}$ is a zero of $\mathbb{Q}_{n}$ and at least one partial derivative of $\mathbb{Q}_{n}$ at $\mathbf{x}^{*}$ is not zero, then we say $\mathbf{x}^{*}$ is a simple zero of $\mathbb{Q}_{n}$.

Corollary 3.4. Under the assumption of Theorem 3.1, the quasi-orthogonal polynomial $\mathbb{Q}_{n}$ can have only simple zeros.

Proof. Since our $\mathscr{L}$ is square positive, $H_{k}$ in (2.4) is a positive definite matrix. From (2.6) it follows that $K_{n}(\mathbf{x}, \mathbf{x})>0$. Let $\mathbf{x}^{*}$ be a zero of $\mathbb{Q}_{n}$. From (3.3) we have

$$
\mathbb{P}_{n-1}^{T}\left(\mathbf{x}^{*}\right) H_{n-1}^{-1} A_{n, i} \partial_{i} \mathbb{Q}_{n}\left(\mathbf{x}^{*}\right)=K_{n}\left(\mathbf{x}^{*}, \mathbf{x}^{*}\right)>0 .
$$

Therefore, $\partial_{i} \mathbb{Q}_{n}\left(\mathbf{x}^{*}\right)$ cannot be zero.

The coefficients $\lambda_{k n}, 1 \leq k \leq N$, in (2.10) are called the cubature weights associated with the $\mathbf{x}_{k n}$. Our next theorem gives explicit formulae for $\lambda_{k n}$.

Theorem 3.5. If the Gaussian cubature (2.10) exists, then the cubature weights are given by

$$
\lambda_{k n}=\left[K_{n}\left(\mathbf{x}_{k n}, \mathbf{x}_{k n}\right)\right]^{-1} .
$$

In particular, $\lambda_{k n}>0$, and

$$
\lambda_{k n}=\left[\mathbb{P}_{n-1}^{T}\left(\mathbf{x}_{k n}\right) H_{n-1}^{-1} \partial_{i} \mathbb{Q}_{n}\left(\mathbf{x}_{k n}\right)\right]^{-1} .
$$

Proof. By definition of $K_{n}(\cdot, \cdot)$ in (2.6), the polynomial $K_{n}\left(\mathbf{x}_{k n}, \cdot\right)$ belongs to $\Pi_{2 n-2}$. Therefore, from the Gaussian cubature formula and Corollary 3.2 we obtain

$$
\mathscr{L}\left[K_{n}\left(\mathbf{x}_{k n}, \cdot\right)\right]=\sum_{j=1}^{n} \lambda_{j n} K_{n}\left(\mathbf{x}_{k n}, \mathbf{x}_{j n}\right)=\lambda_{k n} K_{n}\left(\mathbf{x}_{k n}, \mathbf{x}_{k n}\right) .
$$

On the other hand, by (2.6),

$$
\begin{aligned}
\mathscr{L}\left[K_{n}\left(\mathbf{x}_{k n}, \cdot\right)\right] & =\mathscr{L}\left[\sum_{j=0}^{n-1} \mathbb{P}_{j}^{T}\left(\mathbf{x}_{k n}\right) H_{j}^{-1} \mathbb{P}_{j}(\cdot)\right] \\
& =\sum_{j=0}^{n-1} \mathbb{P}_{j}^{T}\left(\mathbf{x}_{k n}\right) H_{j}^{-1} \mathscr{L}\left(\mathbb{P}_{j}\right)=1
\end{aligned}
$$

by the orthogonality of $\left\{\mathbb{P}_{n}\right\}$ and the fact that $\mathbb{P}_{0}=1$. Therefore, we have proved (3.4). Clearly, $\lambda_{k n}>0$, and (3.5) follows easily from (3.4) and Theorem 3.3.

Remark. The identity (3.4) was proved by Mysovskikh [8], see also Schmid [12]. We include the proof here because it is simple and illustrative. The identity (3.5) is new. The positivity of $\lambda_{k n}$ also follows as a simple consequence of Theorem 2.2 (see [12]).

Corollary 3.6. Let $\lambda_{k n}$ be as in (2.10). Then $\lambda_{k n}=\min \mathscr{L}\left(P^{2}\right)$, where the minimum is over all $P \in \Pi_{n-1}$ subject to the condition $P\left(\mathbf{x}_{k n}\right)=1$.

Proof. Compare (3.4) with (2.7). 
These formulas for $\lambda_{k n}$ should be compared with those for weights in Gaussian quadratures (cf. [1, p. 66]).

\section{LAGRANGE INTERPOLATION}

The Gaussian cubature formulae are interpolatory (cf. $[10,12,15])$, hence their nodes may be taken to solve an interpolation problem. In this section we consider Lagrange interpolation based on the zeros of $\mathbb{Q}_{n}$. Throughout this section we assume that $\mathbb{Q}_{n}$ is defined as in (2.9).

If $\mathbf{x}_{k}, 1 \leq k \leq N$, are distinct real points in $\mathbb{R}^{2}, N=\operatorname{dim} \Pi_{n-1}$, then we can consider the following Lagrange interpolation problem: For any given function $f$, find a polynomial $P$ in $\Pi_{n-1}$ such that

$$
P\left(\mathbf{x}_{k}\right)=f\left(\mathbf{x}_{k}\right), \quad 1 \leq k \leq N .
$$

For general location of the $\mathbf{x}_{k}$ it is known [4] that the Lagrange interpolation problem is not always solvable. It is almost solvable, i.e., it is solvable unless the nodes lie in some special configuration. Moreover, even if the interpolation is solvable, the explicit formula of the interpolating polynomial is in general not known. However, if the $\mathbf{x}_{k}$ 's are the zeros of $\mathbb{Q}_{n}$, we do have a complete solution.

Theorem 4.1. Suppose $\mathbb{Q}_{n}$ has $N=\operatorname{dim} \Pi_{n-1}$ distinct real zeros $\left\{\mathbf{x}_{k n}\right\}_{k=1}^{N}$. Then for any given function $f$, there exists a unique polynomial $L_{n} f$ in $\Pi_{n-1}$ which satisfies

$$
\left(L_{n} f\right)\left(\mathbf{x}_{k n}\right)=f\left(\mathbf{x}_{k n}\right), \quad 1 \leq k \leq N .
$$

Moreover, $L_{n} f$ is given explicitly by

$$
L_{n} f=\sum_{k=1}^{N} f\left(\mathbf{x}_{k n}\right) \lambda_{k n} K_{n}\left(\cdot, \mathbf{x}_{k n}\right),
$$

where $\lambda_{k n}$ and $K_{n}(\cdot, \cdot)$ are as in (2.10) and (2.6).

Proof. From Corollary 3.2 and Theorem 3.5 it is clear that $L_{n} f$ in (4.2) satisfies (4.1). Since $K_{n}\left(\mathbf{x}_{k n}, \cdot\right) \in \Pi_{n-1}$, we have $L_{n} f \in \Pi_{n-1}$. We now prove that the interpolating polynomial is unique. By our assumption and Theorem 2.1, the Gaussian cubature of degree $2 n-2$ based on the zeros of $\mathbb{Q}_{n}$ exists. Suppose there exists another interpolating polynomial $L_{n}^{*} f \in \Pi_{n-1}$ that satisfies (4.1). Then $\left(L_{n} f-L_{n}^{*} f\right)^{2}$ is in $\Pi_{2 n-2}$ and takes the value zero at $\mathbf{x}_{k n}$. By Gaussian cubature,

$$
\mathscr{L}\left[\left(L_{n} f-L_{n}^{*} f\right)^{2}\right]=\sum_{k=1}^{N} \lambda_{k n}\left(L_{n} f-L_{n}^{*} f\right)^{2}\left(\mathbf{x}_{k n}\right)=0 .
$$

Since $\mathscr{L}$ is a square positive linear functional, it follows that $L_{n} f=L_{n}^{*} f$.

The formula (4.2) is comparable to the corresponding univariate interpolating polynomial. The polynomials $l_{k n}=\lambda_{k n} K_{n}\left(\cdot, \mathbf{x}_{k n}\right), 1 \leq k \leq N$, are the fundamental polynomials of the interpolation. They satisfy

$$
l_{k n}\left(\mathbf{x}_{j n}\right)=\delta_{k j} .
$$

The following formula for $l_{k n}$ seems to be interesting. It follows from (3.1) and (3.2). 
Corollary 4.2. Let $\mathbf{x}_{k n}=\left(x_{k n}^{(1)}, x_{k n}^{(2)}\right), \mathbf{x}=\left(x_{1}, x_{2}\right)$. Then

$$
l_{k n}(\mathbf{x})=\frac{\mathbb{P}_{n-1}^{T}\left(\mathbf{x}_{k n}\right) H_{n-1}^{-1} A_{n, i} \mathbb{Q}_{n}(\mathbf{x})}{\mathbb{P}_{n-1}^{T}\left(\mathbf{x}_{k n}\right) H_{n-1}^{-1} A_{n, i} \partial_{i} \mathbb{Q}_{n}\left(\mathbf{x}_{k n}\right)\left(\mathbf{x}_{i}-\mathbf{x}_{k n}^{(i)}\right)} .
$$

We note that this formula is independent of $i$. In the proof of Theorem 4.1 we have used Gaussian cubature. In the univariate theorem it is the other way around, i.e., the Gaussian quadrature is usually proved by integrating the interpolating polynomial. It would be interesting if one could prove the existence of the Gaussian cubature this way.

Next we consider the convergence behavior of $L_{n} f$ to $f$. We assume that $\mathscr{L}$ is expressible in the form of

$$
\mathscr{L}(f)=\int_{\mathbb{R}^{2}} f(\mathbf{x}) w(\mathbf{x}) d \mathbf{x},
$$

where $w$ is a nonnegative function and $\int w(\mathbf{x}) d \mathbf{x}=1$. By $L_{w}^{2}$ we denote the space of Lebesgue measurable functions $f$ on the support set of $w$ for which the norm

$$
\|f\|_{w, 2}=\int|f(\mathbf{x})|^{2} w(\mathbf{x}) d \mathbf{x}
$$

is finite; $C(\Omega)$ denotes the space of continuous functions on a compact set $\Omega$. Let $\left\{\mathbb{P}_{n}\right\}_{n=0}^{\infty}$ be the orthogonal polynomials associated with $\mathscr{L}$, and $S_{n} f=$ $S_{n}(w, f)$ be the $n$th partial sum of the Fourier orthogonal expansion of $f$ in terms of $\left\{\mathbb{P}_{n}\right\}_{n=0}^{\infty}$. Then $S_{n} f \in \Pi_{n-1}$ and

$$
S_{n} f=\sum_{k=0}^{n-1} \mathbb{P}_{k}^{T} H_{k}^{-1} \int \mathbb{P}_{k} f w d \mathbf{x} .
$$

By Bessel's inequality (cf. [17]),

$$
\left\|S_{n} f\right\|_{w, 2} \leq\|f\|_{w, 2} \text {. }
$$

Theorem 4.3. Let $L_{n} f$ be as in Theorem 4.1. Then for any given function $f$,

$$
\left\|L_{n} f\right\|_{w, 2} \leq \max _{1 \leq k \leq N}\left|f\left(\mathbf{x}_{k n}\right)\right|
$$

Proof. First we write

$$
\left\|L_{n} f\right\|_{w, 2}=\sup _{g} \int\left(L_{n} f\right) g w d \mathbf{x},
$$

where the supremum is taken over those $g$ satisfying $\|g\|_{w, 2}=1$. From our assumption and Theorem 2.1, the Gaussian cubature of degree $2 n-2$ exists. Therefore, by orthogonality, the Gaussian cubature (2.10), and the Cauchy inequality, we have

$$
\begin{aligned}
\int\left(L_{n} f\right) g w d \mathbf{x} & =\int\left(L_{n} f\right)\left(S_{n} g\right) w d \mathbf{x}=\sum_{k=1}^{N} \lambda_{k n}\left(L_{n} f\right)\left(\mathbf{x}_{k n}\right)\left(S_{n} g\right)\left(\mathbf{x}_{k n}\right) \\
& \leq\left\{\sum_{k=1}^{N} \lambda_{k n}\left[f\left(\mathbf{x}_{k n}\right)\right]^{2}\right\}^{1 / 2}\left\{\sum_{k=1}^{N} \lambda_{k n}\left[\left(S_{n} g\right)\left(\mathbf{x}_{k n}\right)\right]^{2}\right\}^{1 / 2}
\end{aligned}
$$


Since $\lambda_{k n}>0$ and $\sum_{k=1}^{N} \lambda_{k n}=\mathbf{1}$, where $\mathbf{1}$ is the function taking constant value 1, applying the Gaussian cubature to $\left(S_{n} g\right)^{2}$, we then obtain

$$
\int\left(L_{N} f\right) g w d \mathbf{x} \leq \sup _{1 \leq k \leq N}\left|f\left(\mathbf{x}_{k n}\right)\right|\left\|S_{n} g\right\|_{w, 2} .
$$

The desired result now follows from (4.5) and (4.7).

The idea used in the proof of this theorem goes back to Marcinkiewicz and Zygmund in the context of trigonometric interpolation. This method may also work for $L_{w}^{p}$ convergence, but first $L_{w}^{p}$ convergence of the $S_{n} f$ has to be solved. For the univariate case, see [16] and the references given there. Theorem 4.3 shows that $L_{n} f$ is a bounded operator from $L_{w}^{2}$ to $C(\Omega)$, where $\Omega$ is the smallest compact set that contains all zeros of $\mathbb{Q}_{n} \forall n>0$. In general, the location of $\mathbf{x}_{k n}$ is not known. Even when $w$ has finite support, $\mathbf{x}_{k n}$ may not all be in the support set. This is the reason for our assumptions in the next result.

Corollary 4.4. Let $L_{n} f$ be as in Theorem 4.1, and $W$ denote the support set of $w$. If $\Omega \cup W$ is compact, then

$$
\lim _{n \rightarrow \infty}\left\|L_{n} f-f\right\|_{2, w}=0 \quad \forall f \in C(\Omega \cup W) .
$$

Proof. This is a standard application of the Weierstrass theorem, since for any $P_{n} \in \Pi_{n-1}$,

$$
\begin{aligned}
\left\|L_{n} f-f\right\|_{2, w} & \leq\left\|L_{n}\left(f-P_{n}\right)\right\|_{2, w}+\left\|f-P_{n}\right\|_{2, w} \\
& \leq\left\|f-P_{n}\right\|_{\infty}+\left\|f-P_{n}\right\|_{2, w}
\end{aligned}
$$

by Theorem 4.3 , where $\|\cdot\|_{\infty}$ is taken over $\Omega$.

Finally, we note that our theorems on Lagrange interpolation depend on the assumption that $\mathbb{Q}_{n}$ has $N=\operatorname{dim} \Pi_{n-1}$ distinct real zeros, which is also a necessary and sufficient condition for the existence of Gaussian cubatures of degree $2 n-2$. As we mentioned before, this assumption has been verified only in some special cases (see [7, 13]). We give one example from [7].

Example. For the region $[-1,1]^{2}$ with weight

$$
w(x, y)=\left(1-x^{2}\right)^{1 / 2}\left(1-y^{2}\right)^{1 / 2}
$$

the orthogonal polynomials in $(2.1)$ are

$$
P_{j}^{n}(x, y)=2^{-n} U_{j}(x) U_{n-j}(y),
$$

where the $U_{j}$ 's are the Chebyshev polynomials of the second kind. The components $Q_{j}^{n}, 0 \leq j \leq n$, of $\mathbb{Q}_{n}$ are given by

$$
2^{n} Q_{j}^{n}(x, y)=U_{n-j}(x) U_{j}(y)+U_{j}(x) U_{n-j-1}(y) .
$$

The $\Gamma_{n}$ in (2.9) takes the form $\Gamma_{n}=2 \cdot 4^{-n}\left(E_{n} \mid 0\right)$, where

$$
E_{n}=\left[\begin{array}{lll}
0 & & 1 \\
& . & \\
1 & & 0
\end{array}\right] \text {. }
$$


By means of a trigonometric identity, the common zeros of $Q_{j}^{n}$ are given explicitly in [7]. They are all located inside $[-1,1]^{2}$. Other choices of $\Gamma_{n}$ in (2.9) may also lead to $N$ distinct real zeros of $\mathbb{Q}_{n}$; the complete solution for this weight function is given in [13].

\section{ACKNOWLEDGMENT}

The author thanks Professor H. Berens for stimulating discussion, and Professor H. Schmid for several helpful suggestions and extensive references.

\section{BIBLIOGRAPHY}

1. T. S. Chihara, An introduction to orthogonal polynomials, Math. Appl., vol. 13, Gordon and Breach, New York, 1978.

2. P. J. Davis and P. Rabinowitz, Methods of numerical integration, 2nd ed., Academic Press, New York, 1984.

3. G. Freud, Orthogonal polynomials, Pergamon Press, Oxford, 1971.

4. G. G. Lorentz and R. A. Lorentz, Solvability problems of bivariate interpolation. I, Constr. Approx. 2 (1986), 153-169.

5. H. Möller, Polynomideale und Kubaturformeln, Thesis, Univ. Dortmund, 1973.

6. __ Kubaturformeln mit minimaler Knotenzahl, Numer. Math. 25 (1976), 185-200.

7. C. R. Morrow and T. N. L. Patterson, Construction of algebraic cubature rules using polynomial ideal theory, SIAM J. Numer. Anal. (1978), 953-976.

8. I. P. Mysovskikh, On the construction of cubature formulas with fewest nodes, Soviet Math. 9 (1968), 277-280.

9. __ Numerical characteristics of orthogonal polynomials in two variables, Vestnik Leningrad Univ. Math. 3 (1976), 323-332.

10. _ The approximation of multiple integrals by using interpolatory cubature formulae, Quantitative Approximation (R. A. DeVore and K. Scherer, eds.), Academic Press, New York, 1980, pp. 217-243.

11. H. Schmid, On cubature formulae with a minimal number of knots, Numer. Math. 31 (1978), 282-297.

12. __ Interpolatorische Kubaturformeln, Dissertationes Math. 220 (1983), 1-122.

13. __ On minimal cubature formulae of even degree, Internat. Ser. Numer. Math., vol. 85, Birkhäuser, Basel, 1988, pp. 216-225.

14. Minimal cubature formulae and matrix equation, preprint.

15. A. H. Stroud, Approximate calculation of multiple integrals, Prentice-Hall, Englewood Cliffs, N. J., 1971.

16. Y. Xu, On the Marcinkiewicz-Zygmund inequality, Progress in Approximation Theory (P. Nevai and A. Pinkus, eds.), Academic Press, 1991, pp. 879-891.

17. ___ On multivariate orthogonal polynomials (submitted).

Department of Mathematics and Statistics, University of Arkansas at little Rock, LitTLE Rock, ARkansas 72204

E-mail address: yuan@curie.ualr.edu 